\title{
Dynamic Free-Spectral-Range Measurement for Fiber Resonator Based on Digital-Heterodyne Optical Phase-Locked Loop
}

\author{
Hongchen Jiao ${ }^{*}$, Tao Wang1, Heli Gao ${ }^{1}$, Lishuang Feng ${ }^{2}$, Honghao $\mathrm{Ma}^{2}$ \\ ${ }^{1}$ Institute of Remote Sensing Satellite, China Academy of Space Technology, Beijing, China \\ ${ }^{2}$ Precision Opto-Mechatronics Technology, Key Laboratory of Education Ministry, Beihang University, Beijing, China \\ Email: *jhccast@163.com
}

How to cite this paper: Jiao, H.C., Wang, T., Gao, H.L., Feng, L.S. and Ma, H.H. (2021) Dynamic Free-Spectral-Range Measurement for Fiber Resonator Based on Digital-Heterodyne Optical Phase-Locked Loop. Optics and Photonics Journal, 11, 332-340. https://doi.org/10.4236/opj.2021.118023

Received: June 21, 2021

Accepted: July 31, 2021

Published: August 3, 2021

\begin{abstract}
We propose a novel scheme, based on digital-heterodyne optical phase-locked loop with whole-fiber circuit, to dynamically measure the free-spectral-range of a fiber resonator. The optical phase-locked loop is established with a differential frequency-modulation module consists of a pair of acousto-optic modulators. The resonance-tracking loop is derived with the Pound-Drever-Hall technique for locking the heterodyne frequency of the OPLL on the frequency difference between adjacent resonance modes. A stable locking accuracy of about $7 \times 10^{-9}$ and a dynamic locking accuracy of about $5 \times 10^{-8}$ are achieved with the FSR of $8.155 \mathrm{MHz}$, indicating a bias stability of the resonator fiber optic gyro of about $0.1^{\circ} / \mathrm{h}$ with $10 \mathrm{~Hz}$ bandwidth. In addition, the thermal drift coefficient of the FSR is measured as $0.1 \mathrm{~Hz} /{ }^{\circ} \mathrm{C}$. This shows remarkable potential for realizing advanced optical measurement systems, such as the resonant fiber optic gyro, and so on.
\end{abstract}

\section{Keywords}

Free Spectral Range, Fiber Resonator, Dynamic Measurement,

Digital-Heterodyne Optical Phase-Locked Loop, Resonant Fiber Optic Gyro

\section{Introduction}

Optical resonators have been widely studied and applied in many areas of photonics, such as narrow-linewidth laser sources, optical frequency combs, optical filters, and resonant optic gyroscopes, and so on [1] [2] [3] [4] [5]. An exact description of the free-spectral-range (FSR) is necessary for applications of which the accuracy is related to the measurement of resonant frequencies. As an im- 
portant application, the resonator fiber optic gyros employ the FSR of resonator to estimate the Sagnac frequency shift, and the noise distribution and bias stability of the gyro can be derived by analyzing the FSR drift [6].

The intensity response of the resonator is commonly adopted in FSR measurement techniques [7] [8]. On the other hand, a technique based on a modified PDH error signal has been demonstrated with accuracy of 1 part in $10^{4}$ [9]. Moreover, the optical frequency comb has provided several methods for measuring FSR [10] [11] [12] [13]. Combining an optical frequency comb with a tunable diode laser, a broadband and accurate scheme could be achieved [14]. Additionally, a technique based on a narrow linewidth $(1 \mathrm{KHz})$ laser source has been demonstrated with subhertz accuracy [15], in which a previously demonstrated technique based on the Pound-Drever-Hall (PDH) error signal is improved in accuracy by the use of a narrow linewidth laser swept in frequency via an acousto-optic modulator, or single sideband generation.

Although these methods mentioned above show convenient manners for measuring the FSR, it is a remarkable fact that these methods combining a modulator and a reference interferometer require laser frequency sweeping, where the nonlinearity could introduce systematic measurement error [16].

In this study, we demonstrate a novel scheme for dynamic FSR measurement, which combines a digital-heterodyne optical phase-locked loop (OPLL) based on the whole-fiber circuit with a pair of acousto-optic modulators (AOMs) under differential frequency-modulation mode. The drift of the beat frequency between the two beams in the resonator is reduced about four orders of magnitude, as the OPLL turned on and a stable locking precision of $0.032 \mathrm{~Hz}$ (accuracy of about 7 $\times 10^{-9}$ ) can be achieved. When the locking of two beams to adjacent resonant modes is attained, a dynamic locking precision of $0.4 \mathrm{~Hz}$ (accuracy of about $5 \times$ $\left.10^{-8}\right)$ is achieved. In addition, the thermal drift coefficient of the FSR is measured as $0.1 \mathrm{~Hz} /{ }^{\circ} \mathrm{C}$, which is matched well with the academic result.

\section{Principles and Solutions}

\subsection{Dynamic FSR Measurement Scheme}

As the feedback parameter of the reference DDS in the OPLL, which is severely in proportion to the FSR, is used as the estimation of the FSR, the frequency-modulating nonlinearity and the concomitant power drift of the AOM has little effect on the measuring accuracy. On the other hand, the beat signal is generated by two homologous paths of light (from one laser source) with different frequencies. This indicates a much lower phase noise than that of two independent lasers and releases the demands of the system delay and the loop bandwidth [17]. This scheme is mainly of interest for the requirements of applications demanding dynamic accurate FSR measurement, such as the resonator fiber optic gyro (RFOG) [18].

The structure of our novel scheme for dynamic FSR measurement is shown in

Figure 1. The light from the laser is equally split into two parts by the fiber 


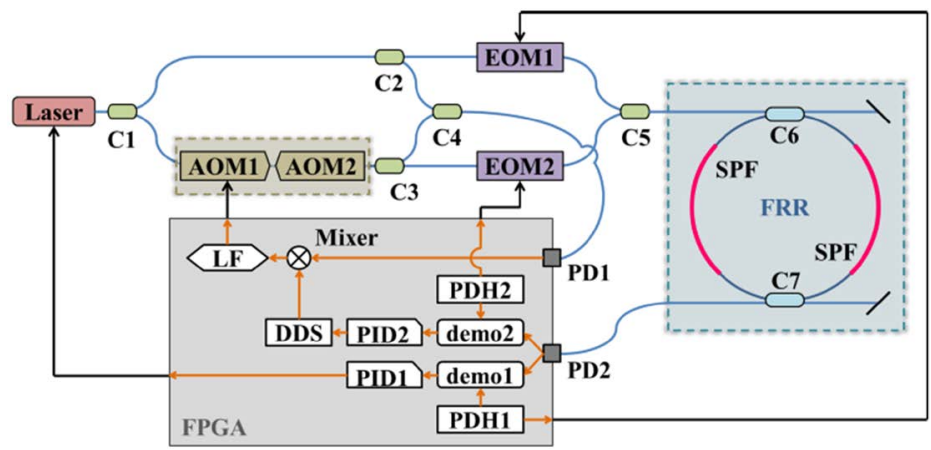

Figure 1. Structure of the novel scheme for dynamic FSR measurement combining a digital-heterodyne OPLL based on a whole-fiber circuit with a pair of AOMs.

coupler $\mathrm{C} 1$. One part of the light is modulated by the electro-optic modulator EOM1 with the signal from PDH1. Another part of the light, whose frequency is shifted by AOM1 and AOM2, is modulated by the electro-optic modulator EOM2 with the signal from PDH2. These two parts of light are both introduced into the fiber ring resonator (FRR) by the fiber coupler $\mathrm{C} 5$ and launched in the photodetector PD2. The signal modulated by EOM1 is demodulated by the demo1, and the demodulation result is treated as the error signal of PID1 to lock the laser frequency on the resonant mode of the FRR. The signal modulated by EOM2 is demodulated by the demo2, and the demodulation result is treated as the error signal of PID2 to lock the frequency of the DDS (equal to the frequency difference between the two parts of light from C1) on one FSR.

Considering that the frequency shift range of a single AOM is about $20 \mathrm{MHz}$ around $80 \mathrm{MHz}$, which is much bigger than the FSR of about $8 \mathrm{MHz}$ (as the length of the FRR is about $25 \mathrm{~m}$ ), we connect the AOM1 and the AOM2 oppositely. Thus, a frequency shift range of about $20 \mathrm{MHz}$ around zero $\mathrm{Hz}$ can be reached. The beat signal, derived by $\mathrm{C} 4$ combining the light from $\mathrm{C} 2$ with the light from C3, is sent to PD1. Next, this signal is mixed with the reference signal of DDS (digital-heterodyne reference of the OPLL), and a feedback signal generated through a Proportional-Integral controller (PI) and a low-pass filter (LF) is used to control the frequency shift of AOM1. In this way, the frequency bias and the phase of the differential frequency-modulation module (DFMM) consists of a pair of AOMs could be lock on these of the DDS. Additionally, two pieces of single polarization fiber (SPF) are fused into the FRR to reduce the effect of undesired polarization light on the resonant modes. In this case, the aberrance of the FSR caused by undesired polarization light could be ignored [19]. Finally, with this novel scheme, a precise measurement of the FSR can be derived.

\subsection{Digital-Heterodyne Optical Phase-Locked Loop}

The digital-heterodyne OPLL module consists of a DFMM, three fiber couplers (C1, C2, and C3), a photodetector (PD1), and a phase discriminator (including the DDS, the Mixer, the PI and the LF). The schematic diagram of this module is shown in Figure 2. In this case, the Photodetector could be treated as an 


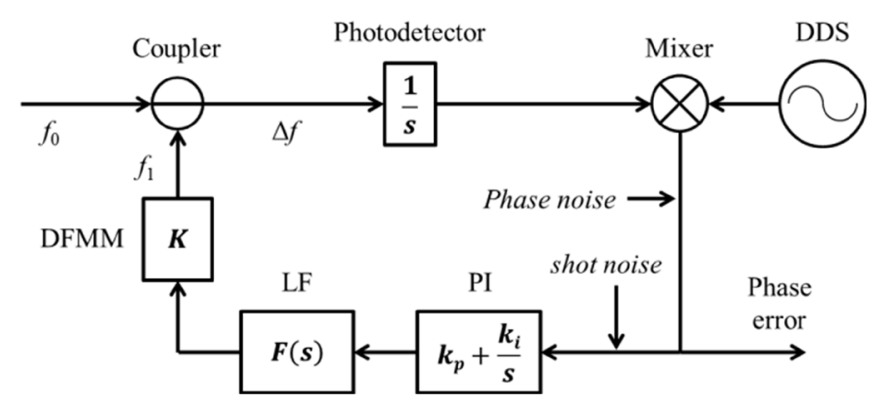

Figure 2. Schematic diagram of the digital-heterodyne OPLL with a whole-fiber circuit based on a DFMM.

integrator. $k_{p}$ is the scale factor of the proportional part of the PI, and $k_{p}$ is the scale factor of the integral part of the PI.

As the OPLL turned on, the phase error would be fluctuating around zero. Thus, the signal sent to the Mixer can be written as

$$
I=I_{0}+I_{1}+2 \sqrt{I_{0} I_{1}} \cos (\Delta \phi) ; \Delta \phi=\int_{0}^{t} \Delta f(\tau) d \tau .
$$

Here, $I_{0}$ is the intensity of the light from C2, $I_{1}$ is the intensity of the light from C3, $t$ is the detection time, $\Delta \phi$ is the phase difference between two paths of light and $\Delta f$ is the frequency difference of the beat signal equal to $f_{1}-f_{0}$. Therefore, the signal $P$ sent to PI can be written as

$$
\begin{aligned}
& P=\sqrt{I_{0} I_{1}} \sin (\delta \phi-\Delta \phi)+\sqrt{I_{0} I_{1}} \sin (\delta \phi+\Delta \phi)+\left(I_{0}+I_{1}\right) \sin (\delta \phi) ; \\
& \delta \phi=\int_{0}^{t} f_{D D S}(\tau) d \tau
\end{aligned} .
$$

Here, $\delta \phi$ is the phase of the DDS, the $f_{D D S}$ is the frequency of the DDS. When the $\Delta f$ is approach to $f_{D D S}$, in the $P$, the spectral distribution of the first term is much lower than that of the second term and that of the third term. As the cut off frequency of the LF is set to be higher than $\left|\Delta f-f_{D D S}\right|$ and lower than $f_{D D S}$, the second term and third term of the LF would be well removed. Additionally, the output of the LF should be a clear signal in direct proportion to the $|\delta \phi-\Delta \phi|$, and this phase difference should be well locked on zero. Whereas, in practical, there are many kinds of noises, other than the fluctuation of the $\Delta \phi$, would be introduced in the OPLL, and the most serious noises are the phase noise of the DDS and the shot noise of the photodetector. The former would be coupled into the phase error directly, and the later would induce a frequency drift of $f_{1}$. The phase error variance for the loop, including phase noise and shot noise is [20]

$$
\begin{aligned}
& \sigma^{2}=\int_{-\infty}^{\infty} S_{p}(f)|1-H(j 2 \pi f)|^{2} d f+\int_{-\infty}^{\infty} \frac{S_{s}(f)}{k_{d}{ }^{2}}|H(j 2 \pi f)|^{2} d f \\
& S_{p}(f)=\delta f /\left(2 \pi f^{2}\right) ; S_{s}(f)=2 e R I_{1} \\
& k_{d}=2 R \sqrt{I_{0} I_{1}}
\end{aligned}
$$

Here, the $S_{p}(f)$ and $S_{s}(f)$ are the double sided power spectra of these two noises, $\delta f$ is the summed full wave at half maximum (FWHM) linewidth of the beat 
signal and the DDS, $R$ is the detector responsivity, $k_{d}$ is the detector gain constant and $H(j 2 \pi f)$ is the closed loop frequency response of the OPLL. In this case, the cut off frequency $f_{c}$ of the LF and the time delay $T_{d}$ of the loop determine the performance of the OPLL.

In our design, the keep-lock time is selected to be 10 years to give reliable operation in a practical system. In this case, the requirement for the $\delta f$ is shown in Figure 3.

In this paper, the tested cut off frequency $f_{c}$ of the LF is about $70 \mathrm{kHz}$, the time delay $T_{d}$ of the loop is about $100 \mathrm{~ns}$. This point is marked in Figure 3 with a black star, where the $\delta f$ should be less than $2.7 \mathrm{kHz}$ to match the requirement for a stable phase-locked state.

\section{Experiments and Conclusions}

In order to verify the efficiency of the novel OPLL, a sinusoidal signal with a frequency of $4.5 \mathrm{MHz}$, replacing the output of the DDS, is used as the heterodyne reference. The spectral distribution of the beat signal, between two paths of light, under unlocked condition and locked condition are both recorded.

We draw out a piece of the test, and put the instantaneous spectral distribution under the unlocked condition at the left face in Figure 4 and the instantaneous spectral distribution under the locked condition at the right face in Figure 4. The inserted face at the mountainside of Figure 4 represents the cut-off height of $20 \mathrm{~dB}$ attenuation from the peak value.

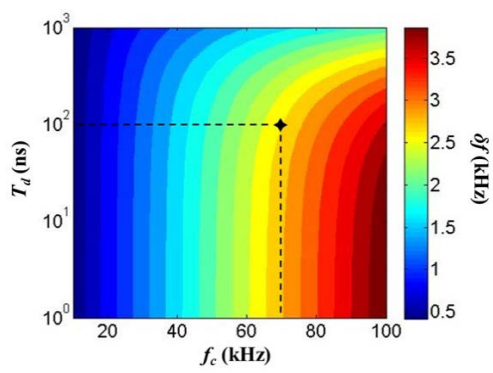

Figure 3. Requirement for the linewidth of the beat signal $\delta f$ with different cut off frequency $f_{c}$ of the LF and time delay $T_{d}$ of the loop.

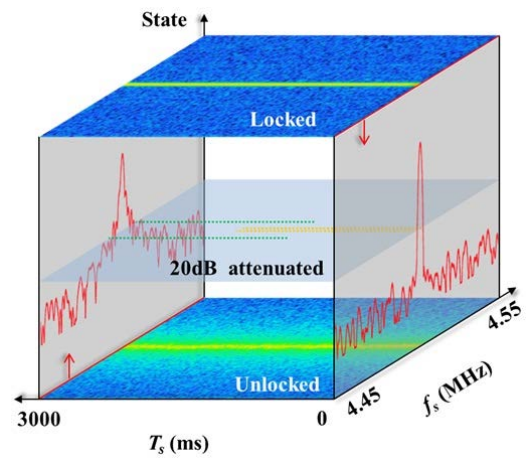

Figure 4. Tests of the spectral distribution of the beat signal between two paths of light (under unlocked condition and locked condition). 
As shown in Figure 4, the linewidth of the beat signal under the locked condition is effectively reduced than that of the beat signal under the unlocked condition, which introduces a centralization of the power of the beat signal. In addition, the drift of the center frequency of the beat signal is well suppressed, meaning that the $\Delta f$ is absolutely controlled by the heterodyne reference of the OPLL.

The power spectral density (PSD) is used to analyze the center-frequency drift of beat signal and the locking precision of the OPLL. As shown in Figure 5, the drift of center-frequency is reduced of about four orders of magnitude and the beat frequency noise within $10 \mathrm{~Hz}$ (considering the application environment of this technology is RFOG, which usually has a bandwidth of about some $\mathrm{Hz}$ ) is about $0.032 \mathrm{~Hz}$, meaning a locking accuracy of about $7 \times 10^{-9}$ under stable heterodyne reference.

According to the structure in Figure 1, an experimental platform for dynamic FSR measurement is constructed as shown in Figure 6. The diameter of the fiber ring is set to $90 \mathrm{~mm}$ to efficiently remove the undesired polarization light with SPFs. The FRR packaged in an acrylic box is fixed on a temperature controlling module (TCM).

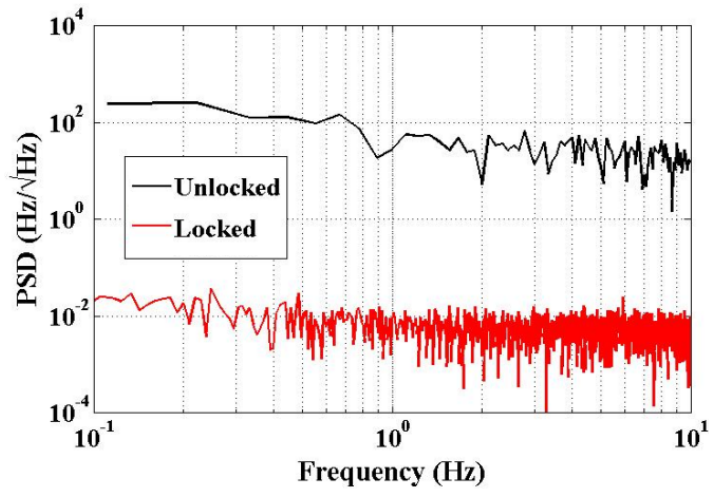

Figure 5. PSDs of the center-frequency drift of beat signals with the OPLL enabled (Locked) and with the OPLL disenabled (unLocked).

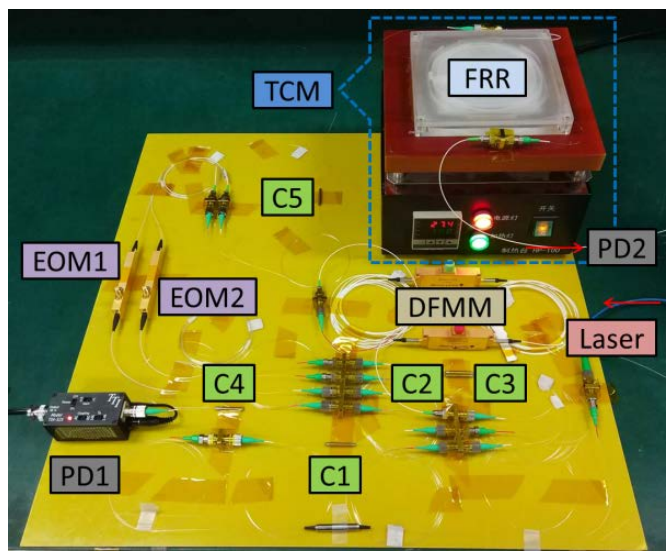

Figure 6. Experimental platform for dynamic FSR measurement based on the novel scheme. 
With this experimental platform, the FSR of the FRR has been tested. The TCM is set to five temperature points, and every state would be held on for 30 min. The averages of the FSR at every state are shown in Figure 7 with green spots. A linear fit has been taken and plotted in Figure 7 with red line, and the thermal drift coefficient $\partial F S R / \partial T$ can be calculated as $0.1 \mathrm{~Hz} /{ }^{\circ} \mathrm{C}$. The drift range of the data is about $0.4 \mathrm{~Hz}$ as shown in Figure 7 with gray dash lines.

Additionally, the standard deviations (STD) of every state are presented in Figure 8 with histogram, and the black spots and dash line give out how the deviation fluctuate with temperature.

As $30^{\circ} \mathrm{C}$ is approach to the environmental temperature, the TCM would jump between on and off, introducing an exacerbation of the STD. When the TCM is set much higher than environmental temperature, the rate of heat dissipation would be increased and the STD would be exacerbated also. Finally, a dynamic locking precision of $0.4 \mathrm{~Hz}$ (accuracy of about $5 \times 10^{-8}$ ) is achieved.

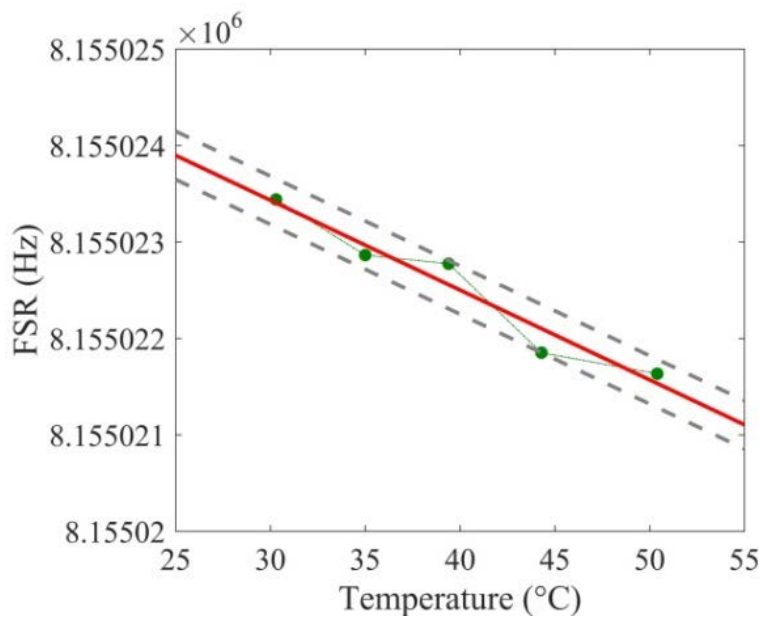

Figure 7. Measurement results (green spots) and linear fit (red line) of FSR of the FRR at different temperature points.

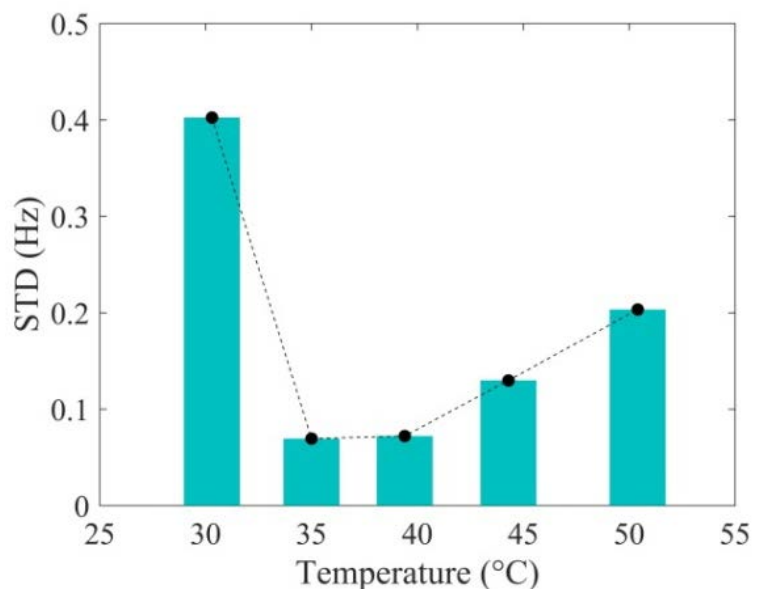

Figure 8. Standard deviations of tested FSR of every state with different temperature points. 


\section{Summary}

In conclusion, we designed and realized a novel scheme for dynamic FSR measurement, which combined a digital-heterodyne OPLL based on the whole-fiber circuit with a DFMM. The transfer function was set up, and the performance characteristics were simulated to optimize the operating stability. With this novel scheme, the drift of center-frequency of the beat signal was reduced about four orders of magnitude. A stable locking accuracy of about $7 \times 10^{-9}$ and a dynamic locking accuracy of about $5 \times 10^{-8}$ were derived. As the FSR was about 8.155 $\mathrm{MHz}$, the drift of the beat frequency would only induce a bias stability of about $0.1^{\circ} / \mathrm{h}$ with a bandwidth of $10 \mathrm{~Hz}$ for the RFOGs.

With better optimized digital-heterodyne OPLL and stable laser source, this potential precision of the RFOG could be further improved. In addition, the thermal drift coefficient of FSR of the FRR $\left(0.1 \mathrm{~Hz} /{ }^{\circ} \mathrm{C}\right)$ was smaller than our normal estimation, which should be caused by the introduction of the polarizing fibers. These would be the next points of interest to us, and the results would be exhibited in our following researches.

\section{Acknowledgements}

Thanks for the funding of National Natural Science Foundation of China (NSFC) (No. 62005015).

\section{Conflicts of Interest}

The authors declare no conflicts of interest regarding the publication of this paper.

\section{References}

[1] Loh, W., Green, A.A.S., Baynes, F.N., Cole, D.C., Quinlan, F.J., Lee, H., Vahala, K.J., Papp, S.B. and Diddams, S.A. (2015) Dual-Microcavity Narrow-Linewidth Brillouin Laser. Optica, 2, 225-232. https://doi.org/10.1364/OPTICA.2.000225

[2] Kippenberg, T.J., Holzwarth, R. and Diddams, S.A. (2011) Microresonator-Based Optical Frequency Combs. Science, 332, 555-559. https://doi.org/10.1126/science.1193968

[3] Tien, M.C. Mizumoto, T., Pintus, P., Kromer, H. and Bowers, J.E. (2011) Silicon ring Isolators with Bonded Nonreciprocal Magneto-Optic Garnets. Opt. Express, 19, 11740-11745. https://doi.org/10.1364/OE.19.011740

[4] Wang, J., Feng, L., Wang, Q., Wang, X. and Jiao, H. (2016) Reduction of Angle Random Walk by In-Phase Triangular Phase Modulation Technique for Resonator Integrated Optic Gyro. Opt. Express, 24, 5463-5468. https://doi.org/10.1364/OE.19.011740

[5] Zhang, J., Ma, H., Li, H. and Jin, Z. (2017) Single-Polarization Fiber-Pigtailed High-Finesse Silica Waveguide Ring Resonator for a Resonant Micro-Optic Gyroscope. Opt. Lett., 42, 3658-3661. https://doi.org/10.1364/OL.42.003658

[6] Feng, L., Wang, J., Zhi, Y., Tang, Y., Wang, Q., Li, H. and Wang, W. (2014) Transmissive Resonator Optic Gyro Based on Silica Waveguide Ring Resonator. Opt. EXpress, 22, 27565-27575. https://doi.org/10.1364/OE.22.027565 
[7] Locke, C.R., Stuart, D., Ivanov, E.N. and Luiten, A.N. (2009) A Simple Technique for Accurate and Complete Characterisation of a Fabry-Perot Cavity. Opt. Express, 17, 21935-21943. https://doi.org/10.1364/OE.17.021935

[8] Yeh, Y., Park, D. and Park, S.H. (2009) High-Speed Measurement of Free Spectral Range Voltage of Tunable Filters. Opt. Lett., 34, 52-54.

https://doi.org/10.1364/OL.34.000052

[9] Gee, S., Ozharar, S., Quinlan, F. and Delfyett, P.J. (2006) High-Precision Measurement of Free Spectral Range of Etalon. Electron. Lett., 42, 715-716. https://doi.org/10.1049/el:20060652

[10] Thorpe, M., Jones, R., Moll, K., Ye, J. and Lalezari, R. (2005) Precise measurements of Optical Cavity Dispersion and Mirror Coating Properties via Femtosecond Combs. Opt. Express, 13, 882-888. https://doi.org/10.1364/OPEX.13.000882

[11] Schliesser, A., Gohle, C., Udem, T. and Hänsch, T.W. (2006) Complete Characterization of a Broadband High-Finesse Cavity Using an Optical Frequency Comb. Opt. Express, 14, 5975-5983. https://doi.org/10.1364/OE.14.005975

[12] Coddington, I., Swann, W.C. and Newbury, N.R. (2008) Coherent Multiheterodyne Spectroscopy Using Stabilized Optical Frequency Combs. Phys. Rev. Lett., 100, Article ID: 013902. https://doi.org/10.1103/PhysRevLett.100.013902

[13] Pupeza, I., Gu, X., Fill, E., Eidam, T., Limpert, J., Tünnermann, A., Krausz, F. and Udem, T. (2010) Highly Sensitive Dispersion Measurement of a High-Power Passive Optical Resonator Using Spatial-Spectral Interferometry. Opt. Express, 18, 26184-26195. https://doi.org/10.1364/OE.18.026184

[14] Del'Haye, P., Arcizet, O., Gorodetsky, M.L., Holzwarth, R. and Kippenberg, T.J. (2009) Frequency Comb Assisted Diode Laser Spectroscopy for Measurement of Microcavity Dispersion. Nat. Photonics, 3, 529-533. https://doi.org/10.1038/nphoton.2009.138

[15] Mandridis, D., Ozdur, I., Bagnell, M. and Delfyett, P.J. (2010) Free Spectral Range Measurement of a Fiberized Fabry-Perot Etalon with Sub-Hz Accuracy. Opt. EXpress, 18, 11264-11269. https://doi.org/10.1364/OE.18.011264

[16] Wang, X., Feng, L.S., Zhou, Z., Li, H., Liu, D.N., Wang, Q.W., Liu, L.Y., Jia, Y.Z., Jiao, H.C. and Liu, N. (2018) Real-Time Free Spectral Range Measurement Based on Optical Single-Sideband Technique. Opt. Express, 26, 7494-7506. https://doi.org/10.1364/OE.26.007494

[17] Gliese, U., Christensen, E.L. and Stubkjaer, K.E. (1991) Laser Linewidth Requirements and Improvements for Coherent Optical Beam Forming Networks in Satellites. IEEE J. Lightwave. Technol., 9, 779-790. https://doi.org/10.1109/50.81982

[18] Wu, J., Smiciklas, M., Strandjord, L.K., Qiu, T., Ho, W. and Sanders, G.A. (2015) Resonator Fiber Optic Gyro with High Backscatter-Error Suppression Using Two Independent Phase-Locked Lasers. Proc. SPIE, 9634, 963410. https://doi.org/10.1117/12.2195220

[19] Jiao, H., Feng, L., Wang, K., Liu, N. and Yang, Z. (2017) Analysis of Polarization Noise in Transmissive Single-Beam-Splitter Resonator Optic Gyro Based on Hollow-Core Photonic-Crystal Fiber. Opt. Express, 25, 27806-27817. https://doi.org/10.1364/OE.25.027806

[20] Ramos, R.T. and Seeds, A.J. (1990) Delay, Linewidth and Bandwidth Limitations in Optical Phase-Locked Loop Design. IEEE Electronics Letters, 26, 389-391. https://doi.org/10.1049/el:19900254 